\title{
Intermittent Theta-burst Stimulation Rescues Dopamine-Dependent Corticostriatal Synaptic Plasticity and Motor Behavior in Experimental Parkinsonism: Possible Role of Glial Activity
}

\author{
Fabrizio Cacace, MSc, ${ }^{1}$ Desirèe Mineo, PhD, ${ }^{1}$ Maria Teresa Viscomi, PhD, ${ }^{1}$ Emanuele Claudio Latagliata, PhD, ${ }^{1}$ \\ Maria Mancini, PhD, ${ }^{1}$ Valeria Sasso, PhD, ${ }^{1}$ Anna Vannelli, PhD, ${ }^{1}$ Tiziana Pascucci, PhD, ${ }^{1,2}$ Valentina Pendolino, PhD, ${ }^{1}$ \\ Elena Marcello, PhD, ${ }^{3}$ Silvia Pelucchi, PhD, ${ }^{3}$ Stefano Puglisi-Allegra, PhD, ${ }^{1,2}$ Marco Molinari, MD, ${ }^{1}$ Barbara Picconi, PhD, ${ }^{1}$ \\ Paolo Calabresi, MD, ${ }^{4}$ and Veronica Ghiglieri, $\mathrm{PhD}^{1,5 *}$ \\ ${ }^{1}$ Fondazione Santa Lucia, Istituto di Ricerca e Clinica a Carattere Scientifico (IRCCS), Rome, Italy \\ ${ }^{2}$ Università di Roma "La Sapienza," Dipartimento di Psicologia, Centro "Daniel Bovet,", Rome, Italy \\ ${ }^{3}$ Dipartimento di Scienze Farmacologiche e Biomolecolari, Università degli Studi di Milano, Milan, Italy \\ ${ }^{4}$ Clinica Neurologica, Dipartimento di Medicina, Università degli Studi di Perugia, Ospedale Santa Maria della Misericordia, S. Andrea delle Fratte, \\ Perugia, Italy \\ ${ }^{5}$ Dipartimento di Filosofia, Scienze Sociali, Umane e della Formazione, Università degli Studi di Perugia, Perugia, Italy
}

\begin{abstract}
Background: Recent studies support the therapeutic utility of repetitive transcranial magnetic stimulation in Parkinson's disease (PD), whose progression is correlated with loss of corticostriatal long-term potentiation and long-term depression. Glial cell activation is also a feature of PD that is gaining increasing attention in the field because astrocytes play a role in chronic neuroinflammatory responses but are also able to manage dopamine (DA) levels.

Methods: Intermittent theta-burst stimulation protocol was applied to study the effect of therapeutic neuromodulation on striatal DA levels measured by means of in vivo microdialysis in 6-hydroxydopamine-hemilesioned rats. Effects on corticostriatal synaptic plasticity were studied through in vitro intracellular and whole-cell patch clamp recordings while stepping test and CatWalk were used to test motor behavior. Immunohistochemical analyses were performed to analyze morphological changes in neurons and glial cells.
\end{abstract}

Results: Acute theta-burst stimulation induced an increase in striatal DA levels in hemiparkinsonian rats, 80 minutes post-treatment, correlated with full recovery of plasticity and amelioration of motor performances. With the same timing, immediate early gene activation was restricted to striatal spiny neurons. Intense astrocytic and microglial responses were also significantly reduced 80 minutes following theta-burst stimulation.

Conclusion: Taken together, these results provide a first glimpse on physiological adaptations that occur in the parkinsonian striatum following intermittent theta-burst stimulation and may help to disclose the real potential of this technique in treating PD and preventing DA replacement therapy-associated disturbances. (c) 2017 International Parkinson and Movement Disorder Society

Key Words: Parkinson's disease; corticostriatal synaptic plasticity; transcranial magnetic stimulation; glia; long-term potentiation
${ }^{*}$ Corresponding author: Dr. Veronica Ghiglieri, Laboratorio di Neurofisiologia, IRCCS Fondazione Santa Lucia, Via del Fosso del Fiorano 64,

00143, Rome, Italy; veronica.ghiglieri@unipg.it

Funding agencies: This work was supported by grants from Fondazione Cariplo (2014-0660 to P.C.) and from the Italian Ministry of Health,

Ricerca Finalizzata, and Giovani Ricercatori (GR-2010-2316671 to V.G., GR-2010.231052 to M.T.V., RF-2013-02357386 to B.P., and RF-201302356215 to P.C.).

Relevant conflicts of interests/financial disclosures: Nothing to report.

Received: 13 November 2016; Revised: 20 February 2017; Accepted: 25 February 2017

Published online 00 Month 2017 in Wiley Online Library (wileyonlinelibrary.com). DOI: 10.1002/mds.26982
In Parkinson's disease (PD), altered dopamine (DA) receptor stimulation exerts its main effect in the striatum, producing changes in input integration, imbalance between direct and indirect striatofugal pathways, and dysfunctional changes in basal ganglia output. As the disease advances, neuronal degeneration also progresses, leading to the emergence of symptoms refractory to conventional therapy.

Increasing evidence suggests a strong relationship between inflammation and nigrostriatal degeneration based on the enhanced vulnerability of nigral neurons 
$\begin{array}{lll}C A C A C E & T H\end{array}$

to oxidative stress, which leads to production of reactive oxygen species, lipid peroxidation, mitochondrial alterations, and neuronal dysfunction. ${ }^{1}$ The resulting propagation of neuroinflammatory responses perpetuates a feed-forward cycle and is considered causative of PD progression. ${ }^{2,3}$ In such a scenario, DA replacement therapy, although it initially alleviates motor symptoms, may bring (lead) to incapability to further respond to neuronal damage and promote cell proliferation and survival.

Repetitive transcranial magnetic stimulation (rTMS) is a noninvasive neuromodulatory technique that allows functional recovery in disease conditions characterized by aberrant forms of synaptic plasticity. ${ }^{4}$ Using theta-burst stimulation (TBS), a specific rTMS pattern, high-frequency bursts of stimuli are delivered at low intensity over cortical areas and produce longlasting inhibitory or facilitatory effects, when continuous (cTBS) or intermittent (iTBS) modes are used, respectively. ${ }^{5}$ The results from clinical studies on the efficacy of TBS in treating PD symptoms are mixed, and no conclusive opinion has been reached so far except for the common evidence that PD patients lack the typical TMS-induced responses. ${ }^{6,7}$ In experimental settings, TBS has different effects in modifying protein expression $^{8}$ and the activity of inhibitory interneurons. ${ }^{9}$ However, neither clinical nor experimental studies have been done in the control condition or PD state to address the specific effect of TBS on corticostriatal plasticity, which is considered an optimal paradigm to study PD-associated synaptic and behavioral alterations. ${ }^{10}$ In PD loss of nigrostriatal terminals, releasing DA is correlated with overt motor symptoms and impairment in the induction of DA-dependent corticostriatal plasticity, that is, long-term potentiation (LTP) and mechanisms of scaling down such as depotentiation and long-term depression (LTD). ${ }^{10,11}$ An interesting feature of rTMS is the ability to induce DA release in the caudate nucleus ${ }^{12}$ and increases in serum DA levels in untreated parkinsonian patients following stimulation. ${ }^{13}$ Similarly, in experimental animals, cortical rTMS is able to induce DA release in subcortical regions ${ }^{14}$ and, in particular, increases the DA content of the dorsolateral striatum. ${ }^{15}$

Based on this evidence, we explored the hypothesis that cortical iTBS may help residual dopaminergic networks to overcome effects of toxic insult, contain neuroinflammation, and rescue striatal plasticity and function in a 6-hydroxydopamine (6-OHDA)-lesioned model of PD.

\section{Methods}

Extended methods may be found in the supplementary data file.

\section{Unilateral 6-OHDA Lesion}

Male Wistar rats $(\mathrm{n}=135)$ for behavior and electrophysiology were deeply anesthetized and unilaterally injected with saline (sham-operated, control (CTRL) $\mathrm{n}=55$ ) or 6-OHDA (6-OHDA-lesioned, $\mathrm{n}=80$ ) into the medial forebrain bundle as previously reported ${ }^{16}$ (Fig. 1a).

\section{Theta-Burst Stimulation}

Sham-operated and 6-OHDA-lesioned rats were subjected to a single iTBS session as previously described ${ }^{17}$ with minor modifications.

\section{Motor Behavior Assessment}

The stepping test was used as previously reported. ${ }^{18}$ Every animal was tested before and 20 or 80 minutes after the exposure to iTBS. Quantitative assessment of gait was obtained in both CTRL and 6-OHDAlesioned prior to stimulation and 80 minutes after the iTBS session using an automated analysis system CatWalk XT (Noldus Information Technology). ${ }^{19}$

\section{Microdialysis}

Rats were anesthetized, mounted in a stereotaxic frame, and implanted with a nonmetal disposable guide cannula (outer diameter of $0.38 \mathrm{~mm}$, length $4 \mathrm{~mm})$ in the striatum ipsilateral to the 6-OHDAlesioned side (anteroposterior $(\mathrm{AP})=-3.6$; mediolateral $(M L)=+1.84)$. Dialysates were collected in awake animals and analyzed as previously reported. ${ }^{20,21}$

\section{Electrophysiology}

Animals receiving sham or real iTBS were divided into two groups, with one sacrificed 20 minutes postiTBS the other sacrificed 80 minutes post-iTBS. Corticostriatal coronal slices were prepared, and glutamatergic excitatory postsynaptic potentials (EPSPs) were evoked in intracellular and whole-cell patch clamp recordings as previously described. ${ }^{16,22}$ LTP and LTD were induced through a high-frequency stimulation (HFS) protocol in magnesium-free and in a physiological medium, respectively. ${ }^{23,24}$

\section{Immunohistochemistry and Confocal Analysis}

Rats were fully anesthetized for perfusion. ${ }^{25}$ The sections were selected and incubated overnight at $4{ }^{\circ} \mathrm{C}$ in phosphate buffer (PB) containing $0.3 \%$ Triton X-100 and $1 \%$ bovine serum albumin (BSA) with primary antibodies then incubated with a cocktail of secondary antibodies as previously described. ${ }^{25}$ To assess lesion, sections including the substantia nigra (SN) and ventral tegmental area were selected and incubated overnight at $4{ }^{\circ} \mathrm{C}$ with a primary antibody rabbit anti-tyrosine hydroxylase (1:500), then with a secondary antibody Alexa Fluor 555 (Thermo Fisher Scientific) donkey 
antirabbit (1:200; Supporting Fig. S1). Confocal image acquisitions and analysis were performed as previously described using a confocal laser-scanning microscope (Zeiss CLSM700, ZEISS Germany). For each section, 2 digital squared frames $\left(\right.$ area $\left.=0.1 \mathrm{~mm}^{2}\right)$ of 6 rostrocaudally spaced sections were examined.

\section{Statistics}

Statistics were performed using Prism 6.0 (GraphPad Software, Inc). Group comparisons were analyzed with 1- or 2-way analysis of variance and with Bonferroni or uncorrected Fisher least significant difference

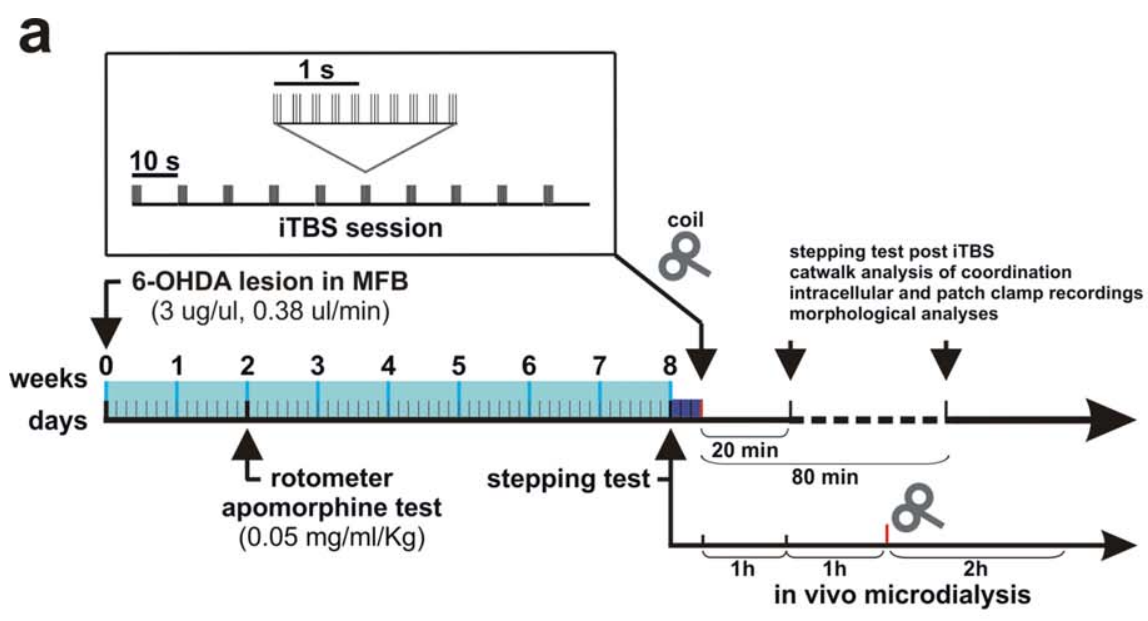

b

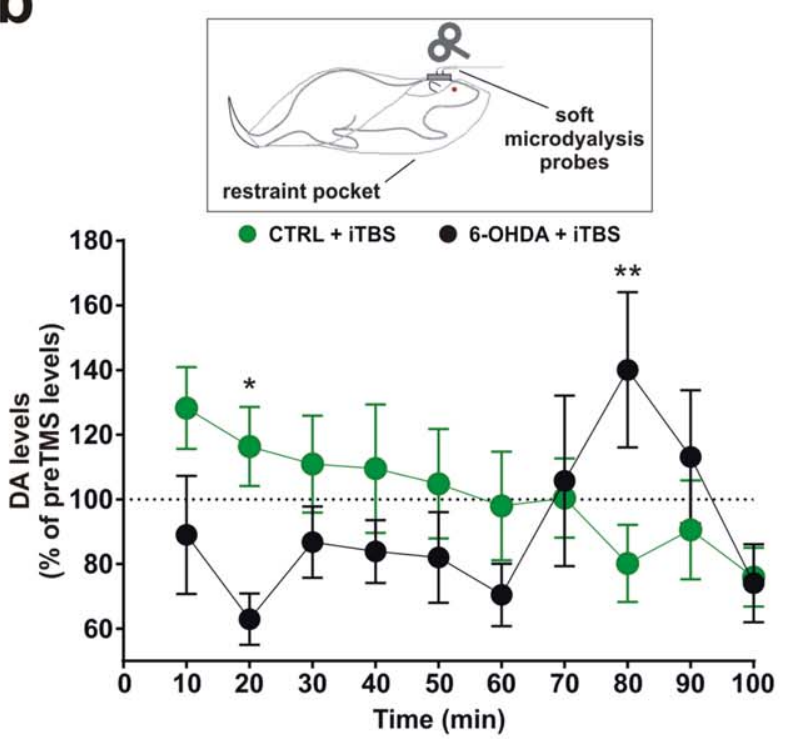

C

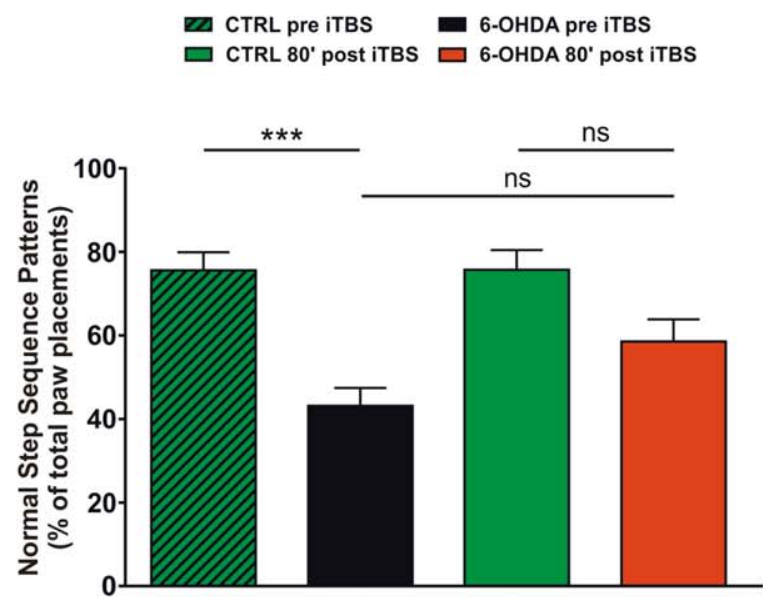

d

6-OHDA 20' post iTBS

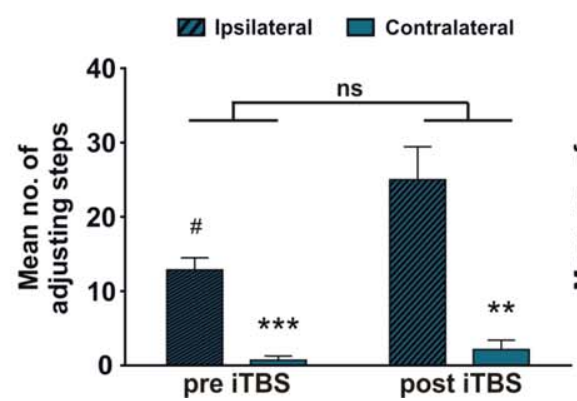

6-OHDA 80' post iTBS

Z Ipsilateral $\square$ Contralateral

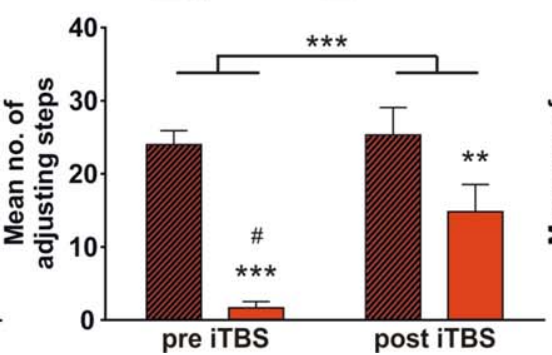

FIG. 1.
6-OHDA $20^{\prime}$ vs $80^{\prime}$

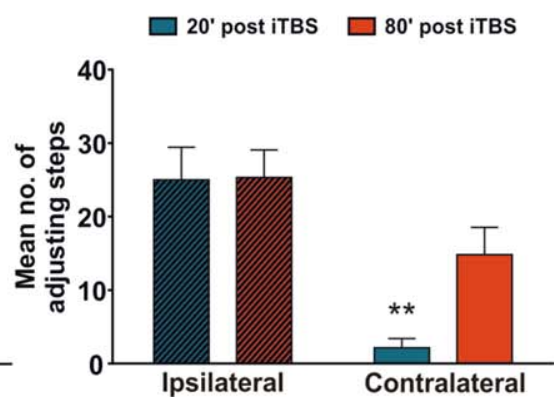


post hoc tests. Values given in the text and in the figures are mean \pm standard error of the mean. A paired Student $t$ test was used to compare pre- versus postHFS protocol in the same cell. An alpha level of .05 was used.

\section{Results}

\section{Acute iTBS Changes DA Levels in Dorsolateral Striatum With a Specific Temporal Pattern}

As extracellular DA levels in both the cortical and subcortical areas seem to be modulated by TMS, 26,27 changes in striatal DA outflow after a single iTBS session were measured by in vivo microdialysis in awake 6-OHDA-lesioned $(\mathrm{n}=7)$ and CTRL $(\mathrm{n}=7)$ rats habituated to a moderate restraint (Fig. $1 \mathrm{~b}$, inset).

DA levels in the CTRL rats were slightly above preiTBS levels with a fast and transient decrease to $-20 \%$ of baseline occurring 80 minutes after stimulation. At the same time, 6-OHDA-lesioned rats showed a peak of DA of approximately $40 \%$ above pre-stimulation baseline while its mean level throughout the other fractions was $15 \%$ below, with a negative peak at 20 minutes. Significant difference was found $20(P<.05)$ and 80 minutes post-treatment $(P<.01$; Fig. $1 \mathrm{~b})$.

\section{iTBS Improves Gait and Akinesia}

To assess changes in the organization of gait in 6OHDA-lesioned and the effects of iTBS, the CatWalk test was used. Although habituated to the experimental setting, hemiparkinsonian rats showed slowness of movements and difficulty in walking through the CatWalk corridor because of their tendency to manifest turning behavior. Therefore, among the variables extracted from the automated analysis, we focused on the regularity index, a sensitive indicator of interpaw coordination. ${ }^{19}$

When subjected to CatWalk trials, hemiparkinsonian rats $(\mathrm{n}=9)$ showed a reduced rate of normal step sequence patterns when compared with the CTRL rats ( $\mathrm{n}=5$; Fig. $1 \mathrm{c} ; P<.001)$. An effect on the regularity index was found in lesioned rats 80 -minutes postiTBS. Although a net difference between pre- and post-iTBS was not reached in the 6-OHDA-lesioned rats, the coordination was more similar between the iTBS-treated groups.

To test the impact of iTBS on asymmetry and forelimb akinesia, a first group of 8 hemilesioned animals was subjected to a stepping test 20 minutes post-stimulation. Although a slight improvement in the impaired forelimb use after iTBS can be noticed (Fig. 1d, left panel; $P<.001$ pre-iTBS comparisons between ipsilateral and contralateral limbs; $P<.01$ post-iTBS, $\mathrm{n}=7$ ), no statistical difference was found between left/right ratio pre- and post-iTBS treatment. This effect was a result of the iTBS-induced general increase of locomotion, significant in ipsilateral limbs $(P<.05)$, which failed to return any significant effect on asymmetry improvement.

A group of 7 hemiparkinsonian animals were tested 80 minutes post-iTBS (Fig. 1d, center panel) and displayed a significant improvement in the impaired forelimb use $(P<.05)$, with a difference in left/right ratio forelimb use $(P<.001, \mathrm{n}=6)$. Overall, the therapeutic effects of iTBS appeared to be time dependent: hemiparkinsonian animals, whose motor activity was tested 80 minutes post-iTBS, showed a higher number of adjusting steps with the impaired forelimb compared to 6-OHDA-lesioned tested 20 minutes after stimulation (Fig. 1d, right panel; $P<.01$ ).

FIG. 1. The intermittent theta-burst stimulation (iTBS) driven dopamine boost affects motor coordination. a: Experimental planning. Animals were subjected to an apomorphine-induced rotation test 2 weeks after receiving intracerebral 6-hydroxydopamine (6-OHDA) or saline. After 6 weeks, the animals were exposed to a single iTBS session and then underwent behavioral tests, electrophysiological recordings, and morphological analyses 20 or 80 minutes after stimulation. b: Time course of in vivo microdialysis performed in dorsolateral striatum showing average DA levels expressed in percentage relative to baseline values (dotted line). Dialysates were collected every 10 minutes starting 1 hour before iTBS application. A time$\times$ treatment interaction was found ( 2 -way analysis of variance: interaction, $F_{9,108}=3.49, P<.001$ ), pointing to an overall difference in their time courses. Statistical difference between the 2 groups ( $n=7$ for both) was revealed 20 (uncorrected Fisher's least significant difference post hoc test, $\left.{ }^{\star} P<.05\right)$ and 80 minutes $\left({ }^{\star \star} P<.01\right)$ after stimulation. c: Histograms showing averaged normal step sequence patterns relative to total number of paw placement calculated as regularity index using CatWalk automated analysis of gait, 80 minutes after iTBS. Although stimulation did not alter the normal step sequence patterns of healthy animals $(n=5)$, DA-depleted animals showed a significant impairment of interpaw coordination ( $n=9$; 1 -way analysis of variance: interaction, $F_{3,64}=11.41, P<.001 ; 6-O H D A$ pre-iTBS vs control (CTRL) pre-iTBS: Bonferroni post hoc, $\left.{ }^{\star \star \star} P<.001\right)$. Acute iTBS treatment did not affect motor activity in CTRL while improving interpaw coordination in lesioned rats, displaying performances similar to healthy controls (6-OHDA 80 minutes iTBS vs CTRL 80 minutes post-iTBS: Bonferroni post hoc, $P>.05$ ). d: Histograms showing mean number of adjusting forelimb steps during stepping test in 6-OHDA-lesioned animals prior to iTBS and $20(n=7)$ or 80 minutes $(n=6)$ later. Left panel: Cortical iTBS induced a general increase of locomotion in both limbs ipsilateral and contralateral to lesion side, but failed to return any significant effect on asymmetry improvement when measured 20 minutes after stimulation (paired Student $t$ test, pre-iTBS, ipsilateral (Ipsi) vs contralateral (Contra): $t_{6}=7.99$, ${ }^{\star \star \star} P<.001$; post-iTBS, Ipsi vs Contra: $t_{6}=5.94$, ${ }^{\star \star} P<.01$; Ipsi, pre-iTBS vs post-iTBS: $t_{6}=3.32$, $\left.{ }^{\#} P<.05\right)$. No statistical difference was found between left/right ratio pre- and post-iTBS treatment (unpaired Student $t$ test, Ipsi/Contra pre-iTBS vs Ipsi/Contra post-iTBS: $t_{14}=1$, $P>.05$ ). Middle panel: Graph showing a significant improvement in the impaired forelimb (contralateral) use was found at 80 minutes (paired Student $t$ test, pre-iTBS, Ipsi vs Contra post hoc, ${ }^{\star \star \star} P<.001$; post-iTBS, Ipsi vs Contra ${ }^{\star \star} P<.01$; Contra, pre-iTBS vs post-iTBS: $t_{5}=3.73$, ${ }^{\#} P<.05$ ). At this time point, a statistical difference in left/right ratio forelimb use was found (unpaired Student $t$ test, Ipsi/Contra pre-iTBS vs Ipsi/Contra post-iTBS: $t_{10}=5.31$, ${ }^{\star \star \star} P<.001$ ). Right panel: Histogram of the time dependence of iTBS effects on the impaired forelimb use resulting in a higher number of adjusting steps performed 80 minutes after stimulation (unpaired Student $t$ test, Contra 20 minutes post-iTBS vs Contra 80 minutes post-iTBS: $t_{11}=3.46$, ${ }^{\star \star} P<.01$ ). [Color figure can be viewed at wileyonlinelibrary.com] 
a
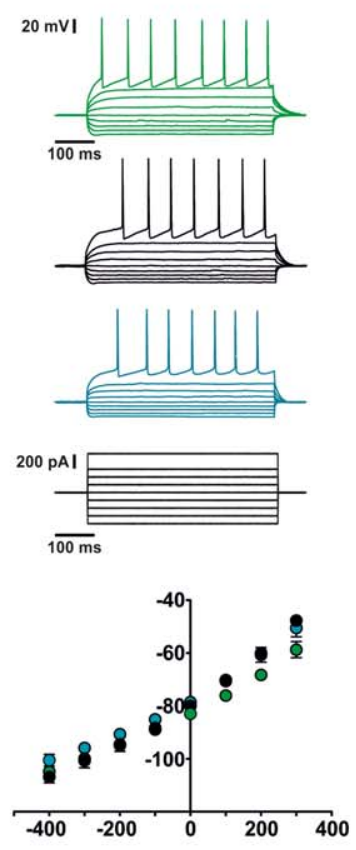

b

LTD protocol
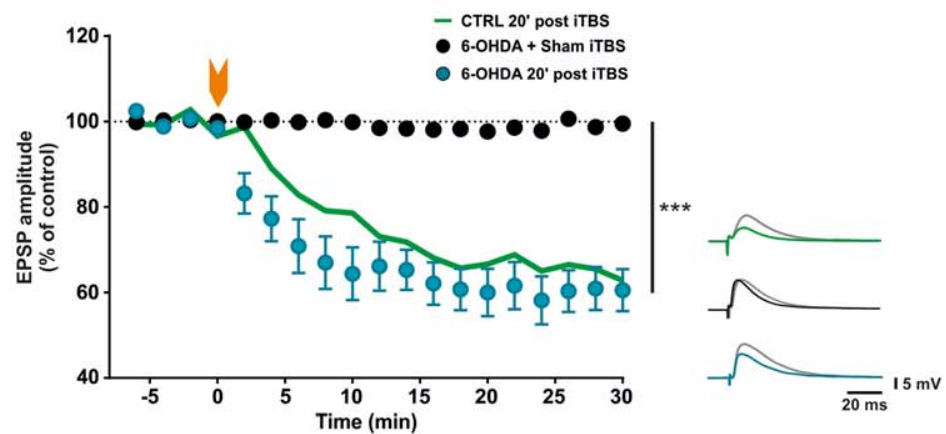

C
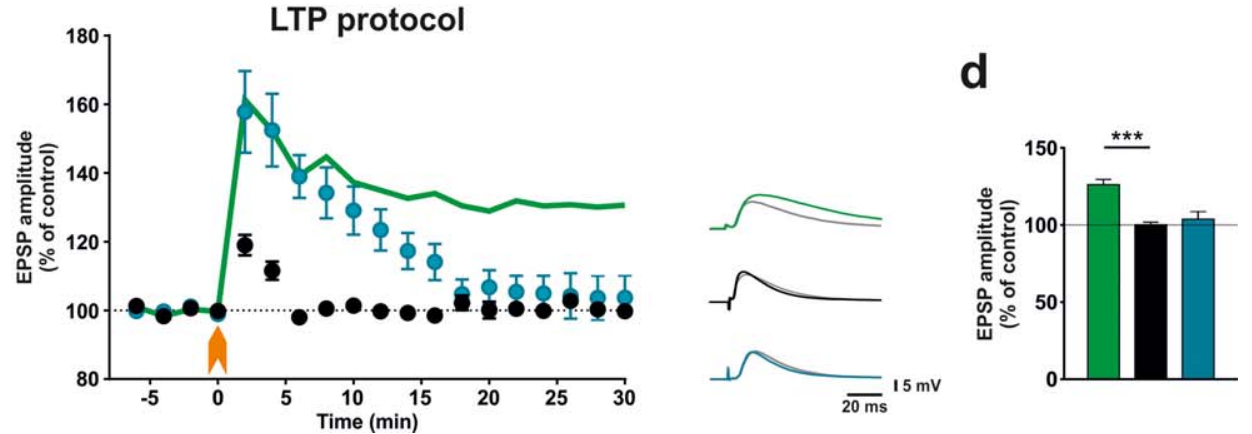

FIG. 2. Corticostriatal synaptic plasticity changes occurring 20 minutes after intermittent theta-burst stimulation (iTBS) stimulation. a, upper panel: Representative current-voltage (I/V) traces of CTRL 20 minutes post-iTBS, 6-hydroxydopamine (6-OHDA)+sham-iTBS and 6-OHDA 20 minutes post-iTBS obtained from striatal projection neurons. Lower panel: Graph showing I/V relationships in the 3 groups. b, upper panel: Time course of excitatory postsynaptic potential (EPSP) amplitude of spiny projection neurons (SPNs) from control (CTRL) exposed to iTBS ( $\mathrm{n}=12$ ) and 6-OHDA-lesioned rats exposed to either sham or real iTBS ( $\mathrm{n}=12$ each group) expressed in percentage of pre-high frequency stimulation (HFS) baseline, measured before and after application of the long-term depression (LTD) protocol. On the right, representative traces of EPSP prior to HFS (gray line) and 30 minutes after HFS (different color per group) are shown. Scale factor is $20 \mathrm{~ms} / 5 \mathrm{mV}$ for all traces. Orange arrow represents HFS protocol. Two-way analysis of variance: interaction, $F_{36,594}=12.01, P<.001$; 6-OHDA 20 minutes post-iTBS $(n=12)$ versus $6-O H D A+s h a m-i T B S(n=12), 30$ minutes after HFS: Bonferroni post hoc, ${ }^{\star \star \star} P<.001$. Lower panel: Time course of EPSP amplitude of SPNs from CTRL exposed to iTBS $(n=11)$ and 6 -OHDA-lesioned rats exposed to either sham or real iTBS ( $n=11$ and $n=10$, respectively) after induction of long-term potentiation (LTP) protocol and representative traces prior to HFS (gray line) and 30 minutes after HFS (different color per group). Two-way analysis of variance: interaction, $F_{36,468}=7.35, P<.001 ; 6-\mathrm{OHDA} 20$ minutes post-iTBS ( $=10$ ) versus $6-O H D A+s h a m-i T B S(n=11)$ : Bonferroni post hoc, $P>.0530$ minutes post-HFS. c: Histogram showing EPSP amplitude 30 minutes post-HFS after LTD protocol (CTRL 20 minutes post-iTBS vs 6 -OHDA+sham-iTBS, ${ }^{\star \star \star} P<.001 ; 6-O H D A 20$ minutes post-iTBS vs $6-O H D A+$ sham-iTBS, $\left.{ }^{\star * \star} P<.001\right)$. d: Histogram of EPSP amplitude 30 minutes post HFS after LTP protocol (CTRL 20 minutes post-iTBS vs $6-$ OHDA+sham-iTBS, ${ }^{\star \star \star} P<.001 ; 6-O H D A 20$ minutes post-iTBS vs $6-\mathrm{OHDA}+$ sham-iTBS, $P>$.05). [Color figure can be viewed at wileyonlinelibrary.com]

No significant effect of iTBS was observed in the CTRL groups $(20$ minutes post-iTBS, $\mathrm{n}=5 ; 80$ minutes post-iTBS, $\mathrm{n}=5$; data not shown). For the stepping test, 1 animal per group was discarded for behavioral alterations during the pre-TMS session that prevented the accomplishment of procedures.

\section{Corticostriatal LTD, but not LTP, Is Rescued 20 Minutes After iTBS}

The effects of iTBS were evaluated comparing neurons recorded from 286 -OHDA-lesioned rats 20 minutes post real or sham-iTBS and from and 18 CTRL rats exposed to real iTBS. In standard artificial CerebroSpinal Fluid (aCSF), firing rate, firing pattern, and current-voltage relationships showed no differences among groups (Fig. 2a). As expected, HFS of corticostriatal fibers failed to induce LTD in striatal projection neurons (SPNs) of 6OHDA-lesioned exposed to sham-iTBS $(n=12)$. In contrast, acute iTBS was able to restore this form of plasticity in the majority of neurons recorded from 6-OHDA- lesioned rats $(n=12)$. In rats exposed to iTBS, once induced, the time course of LTD was similar between 6OHDA and CTRL (Fig. 2b, upper panel).

The ability to induce LTP in a $\mathrm{Mg}^{2+}$-free medium was then tested. In this condition, HFS of corticostriatal fibers induced a LTP in iTBS-treated CTRL rats. Conversely, most of the corticostriatal SPNs recorded from 6-OHDA-lesioned rats exposed to sham-iTBS failed to show long-term changes. Acute iTBS induced in SPNs a potentiation that did not last over time $(n=10)$, with EPSP amplitudes rapidly decreasing to baseline levels (Fig. 2b, lower panel). Baseline EPSPs properties were unchanged by prior exposure to iTBS (Fig. 2b, left, gray). The effects of iTBS on corticostriatal plasticity are summarized in Figures $2 \mathrm{c}$ and $2 \mathrm{~d}$.

\section{LTD and LTP Are Both Completely Restored 80 Minutes After iTBS}

By shifting the sacrifice to 80 minutes post-iTBS, we were able to investigate the effects on corticostriatal 
C A C A C E ET AL

a
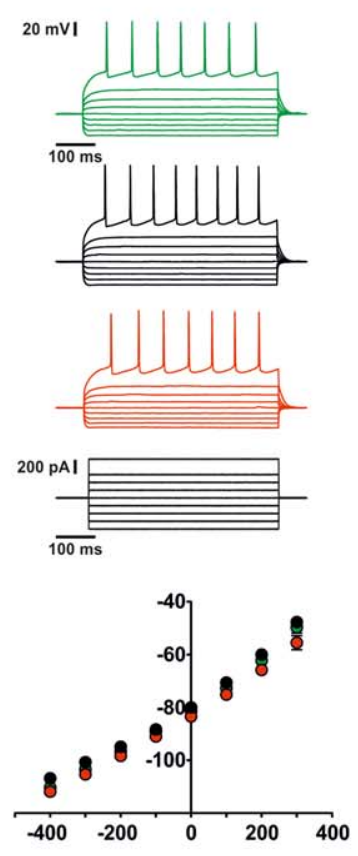

b

\section{LTD protocol}

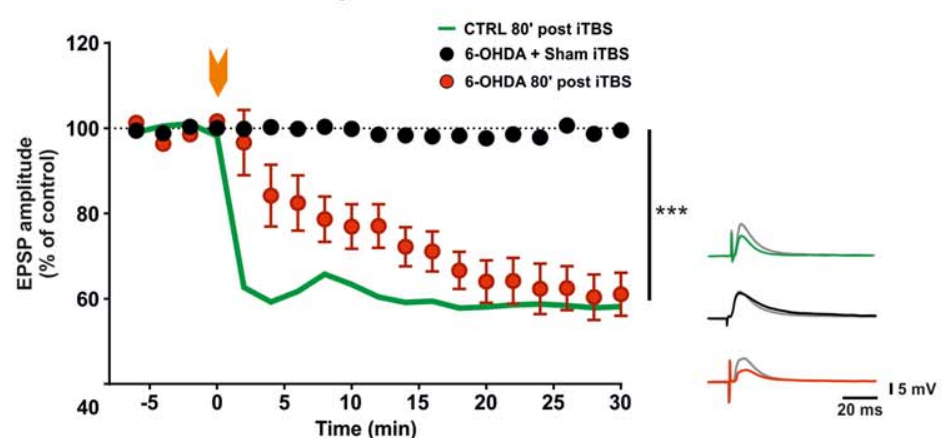

C

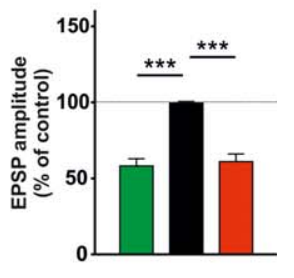

d
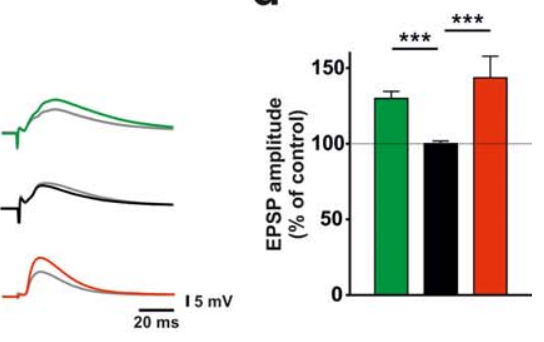

FIG. 3. Corticostriatal synaptic plasticity changes occurring 80 minutes after intermittent theta-burst stimulation (iTBS). a, upper panel: Representative I/V traces of CTRL 80 minutes post-iTBS, 6-hydroxydopamine (6-OHDA)+sham-iTBS and 6-OHDA 80 minutes post-iTBS obtained from striatal projection neurons. Lower panel: Graph showing I/V relationships of the 3 groups. b, upper panel: Time course of excitatory postsynaptic potential (EPSP) amplitude expressed in percentage of pre-high frequency stimulation (HFS) baseline after application of the long-term depression (LTD) protocol. On the right, representative traces of EPSP prior to HFS (gray line) and 30 minutes after HFS (different color per group) are shown. Scale factor is $20 \mathrm{~ms} / 5 \mathrm{mV}$ for all traces. Orange arrow represents the application of HFS protocol. Two-way analysis of variance: interaction, $F_{36,594}=15.22$, $P<.001$; 6-OHDA 80 minutes post-iTBS $(n=12)$ vs $6-O H D A+$ sham-iTBS $(n=12)$ : Bonferroni post hoc, ${ }^{\star \star \star} P<.00130$ minutes post-HFS. Lower panel: Time course of EPSP amplitude expressed in percentage of pre-HFS baseline in long-term potentiation (LTP) protocol and representative traces. Treated 6-OHDA rats recover a physiological LTP 80 minutes after iTBS. Two-way analysis of variance: interaction, $F_{36,486}=4.35, P<.001 ; 6-O H D A$ 80 minutes post-iTBS $(n=11)$ versus $6-O H D A+$ sham-iTBS $(n=11)$ : Bonferroni post hoc, ${ }^{\star \star} P<.00130$ minutes post-HFS. c: Histogram showing comparison of EPSP amplitude 30 minutes post-HFS in LTD protocol (CTRL 80 minutes post-iTBS [n=12] vs 6-OHDA+sham-iTBS, ${ }^{\star \star \star} P<.001$; 6OHDA 80 minutes post-iTBS vs $6-\mathrm{OHDA}+$ sham-iTBS, $\left.{ }^{\star \star \star} P<.001\right)$. d: Histogram showing mean percentage of EPSP amplitude 30 minutes post-HFS in LTP protocol (CTRL 80 minutes post-iTBS [ $=11$ ] vs $6-O H D A+s h a m-i T B S,{ }^{\star \star \star} P<.001 ; 6-O H D A 80$ minutes post-iTBS vs $6-$ OHDA+sham-iTBS, $\left.{ }^{\star \star \star} P<.001\right)$. [Color figure can be viewed at wileyonlinelibrary.com]

plasticity of 11 6-OHDA-lesioned rats and 8 CTRL rats at a time point corresponding to the DA peak revealed by microdialysis measurements. At this time, intrinsic membrane properties were found unaffected (Fig. 3a). Similarly, LTD appeared to be restored in 6-OHDA-lesioned rats exposed to iTBS when compared with animals subjected to sham-iTBS $(\mathrm{n}=12$, $P<.001)$. Both LTD induction and maintenance were not influenced by the DA peak as there was no statistical difference between 6-OHDA rats 80 minutes post-iTBS and CTRL rats 80 minutes post-iTBS (Fig. $3 \mathrm{~b}$, upper panel). Conversely, in the SPNs of hemilesioned rats, the ability to induce and maintain corticostriatal LTP was fully recovered by iTBS (Fig. 3b, lower panel; $\mathrm{n}=11, P<.001)$. Similar to the protocol at 20 minutes, baseline EPSP properties appeared unchanged by iTBS when tested 80 minutes post-iTBS (Fig. 3b, gray). Changes in corticostriatal plasticity expressed in percentages are summarized in Figure $3 \mathrm{c}$ and $\mathrm{d}$.

\section{c-Fos Is Selectively Activated in Spiny Neurons After iTBS}

Qualitative and quantitative changes of c-Fos activation after iTBS were analyzed in the motor cortex, dorsal striatum, $\mathrm{SN}$, and motor thalamus of lesioned $(\mathrm{n}=15)$ and CTRL rats $(\mathrm{n}=15)$ by confocal microscopy techniques (12 samples/rat; $\mathrm{n}=5$ animals/group). In the sham-iTBS-exposed CTRL group, in all brain areas analyzed, c-Fos immunostaining pattern was characterized by a widespread and faint cellular immunopositivity with some scant c-Fos+/DARPP$32+$ cells (data not shown). After 20 minutes and 80 minutes post-iTBS, no significant changes were observed in the motor cortex, $\mathrm{SN}$, or thalamus (data not shown) or in the dorsolateral striatum (Fig. 4a,c) compared to sham-iTBS. In the striatum of 6-OHDA rats exposed to sham-iTBS, the c-Fos expression pattern (Fig. 4a) and the total number of c-Fos + cells were not significantly different when compared with the CTRL rats (Fig. 4c, upper panel). When 
hemiparkinsonian animals were exposed to iTBS and sacrificed 20 minutes later, a slight increase of total number of c-Fos + cells was found in the striatum (Fig. 4c, upper panel). However, by shifting the sacrifice to 80 minutes, we observed a significant effect on striatal c-Fos activation (Fig. 4c, upper panel; $P<.001)$. Double immunofluorescence of $c$-Fos and DARPP-32 confirmed that c-Fos was mainly expressed by striatal SPNs (Fig. 4c, lower panel; $P<.001$ ). To determine the specificity of this effect, the double labeling of c-Fos and choline acetyltransferase or parvalbumin was performed showing lack of c-Fos activation (Fig. 4b). Furthermore, this effect was exclusively restricted to the striatum as c-Fos expression was not enhanced in the motor cortex, SN, or thalamus (Supporting Fig. S2).

\section{iTBS Reduces Astrogliosis and Microglial Activation}

To characterize the effects of TMS treatment on glial responses induced by 6-OHDA lesion, astrocytes and microglia were analyzed by confocal microscopy (12 samples/rat; $\mathrm{n}=5$ animals/group). In sham-iTBS treated CTRL rats, the number of Glial Fibrillary, Acidic, Protein (GFAP) + astrocytes and of ionized calcium binding adaptor molecule 1 (Iba-1) + microglial cells was very low (data not shown). At 20 or 80 minutes post-iTBS, the total number of GFAP + astrocytes and of Iba- $1+$ microglial cells were not changed in the CTRL groups subjected to stimulation (Fig. 5b). An intense astrocytic response was observed in the striatum ipsilateral to the lesion (Fig. 5a). In 6-OHDA+sham-iTBS animals, many densely stained and hypertrophic GFAP + cells with long and ramified processes were found (Fig. 5b, upper panel; $P<.001)$. Similarly, a great number of Iba$1+$ activated microglial cells was found (Fig. 5b, lower panel; $P<.001$ ). When sacrificed 20 minutes post-iTBS, a slight, but not significant, decrease of the total number of GFAP + and Iba- $1+$ cells was observed (Fig. $5 a, b)$. By shifting the sacrifice to 80 minutes, a significant reduction of the total number of GFAP + and Iba$1+$ cells (Fig. 5a,b) compared to all conditions was found $(P<.001)$.

\section{Discussion}

With this study, we bring evidence of the possible role of glial cells in mediating the TMS-dependent recovery of striatal functions in experimental parkinsonism. Acute exposure to iTBS protocol caused an increase in striatal levels of DA, ipsilateral to 6OHDA lesion, at a specific time point corresponding to 80 minutes after stimulation. Concurrently, the ability to express DA-dependent bidirectional plasticity, recorded in SPNs, was recovered and associated with a significant increase in region- and cell typespecific c-Fos immunoreactivity. Behavioral correlates were a major decrease of forelimb akinesia and improvement of gait, supporting the idea that a rescue of corticostriatal plasticity may underlie recovery of motor control. A restoration of plasticity was associated with widespread effects in the dorsolateral striatum, showing marked reduction of both astrogliosis and abnormal microglia activation.

In PD therapy, the use of rTMS has considerably increased during the past decade owing to the evidence that neurostimulation can induce DA release in the caudate nucleus of healthy subjects when elicited on prefrontal ${ }^{28}$ and motor cortical areas. ${ }^{12}$ Based on these clinical findings and on the observation that rTMS is able to induce DA release in subcortical regions of experimental animals, ${ }^{14,15,26}$ we asked whether iTBS, a protocol known to induce longlasting activation of cortical areas, ${ }^{29}$ could overcome the effects of denervation and rescue a physiological synaptic activity by reorganizing cortico-subcortical networks. We previously demonstrated that 20 minutes after a single session of iTBS, a moderate recovery of corticostriatal LTD, which is lost in the PD condition, was associated with a net increase of excitability in all of the striatal neuronal populations, measured as excitatory postsynaptic field potentials through extracellular recordings. ${ }^{17}$ This first observation provided the basis for exploring the hypothesis that a rearrangement of cortical networks together with increased striatal DA levels could possibly recover corticostriatal plasticity at a single cell level.

Here, we explored the time dependence of iTBS effects on parkinsonism and linked the increase in striatal DA levels produced by iTBS to the rescue of bidirectional corticostriatal plasticity and striatal functions. To measure DA variations, we employed intracerebral guide cannulas and probes compatible with magnetic field that allow in vivo microdialysis measurements with minimal tissue damage. Previous work showing TMS-induced changes in DA levels and release were performed in healthy animals using different patterns of stimulation. ${ }^{15}$ Although in our model rats show nearly full lesion, which could possibly have made it difficult to detect striatal DA, we could monitor DA concentration changes in the dorsolateral striatum after iTBS exposure. Notably, most of the animals displayed a distinctive and consistent time course characterized by a peak around 80 minutes post-stimulation.

To find a behavioral correlate of these changes, we used two tests that provided motor activity information rapidly and, shortly after, prepared the brains for electrophysiological and immunohistochemical analyses within the same time point. At 20 minutes, little or no effect of iTBS was observed in counteracting the 
CACACE ET AL)

a
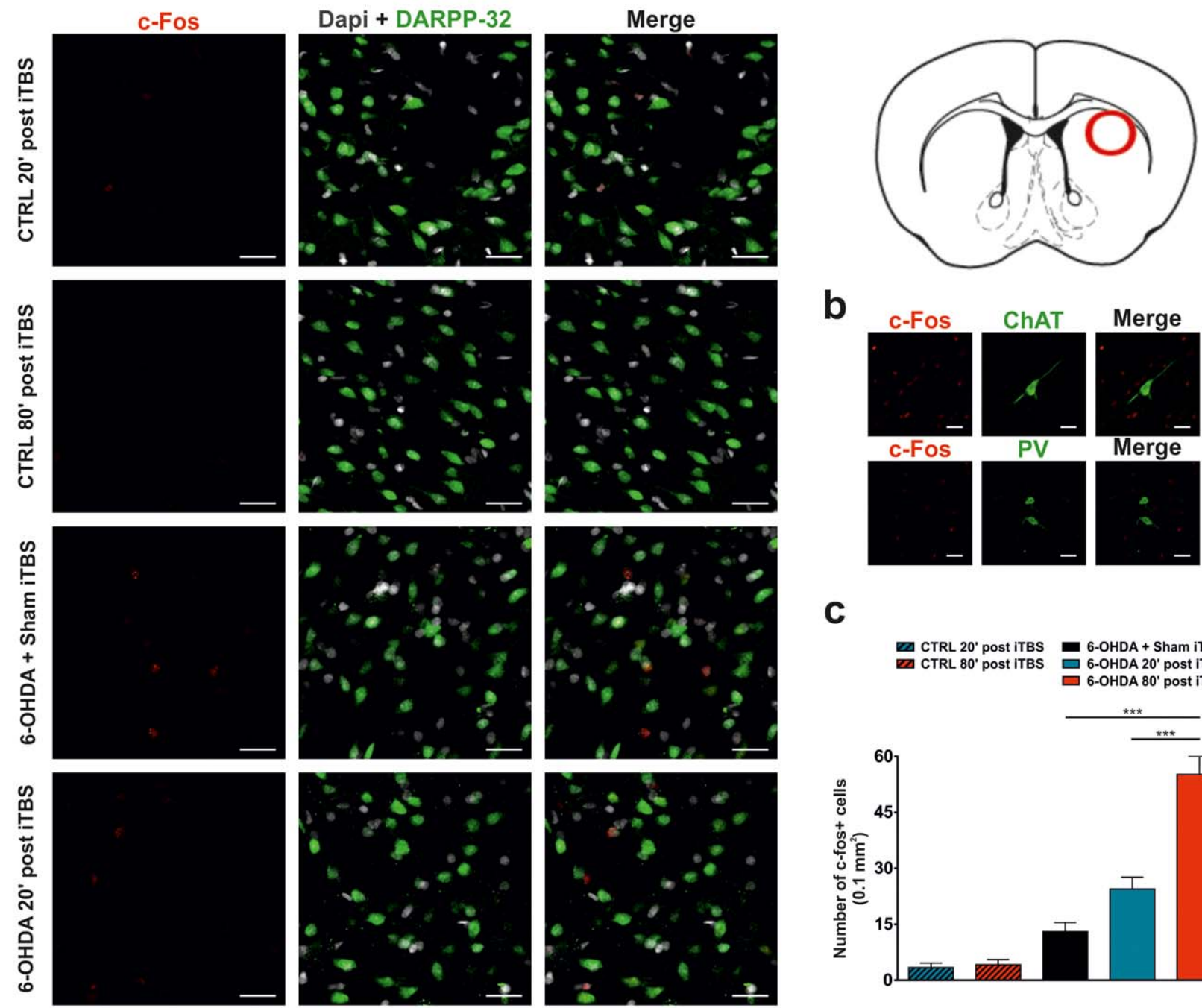

b
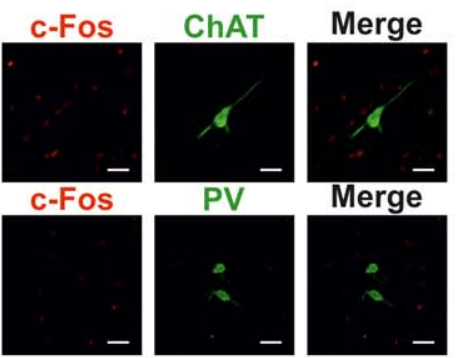

Merge

C

Z CTRL 20' post iTBS 6-OHDA + Sham iTBS Z CTRL 80' post iTBS $\square$ 6-OHDA 20' post ITBS $\square$ 6-OHDA 80' post iTBS
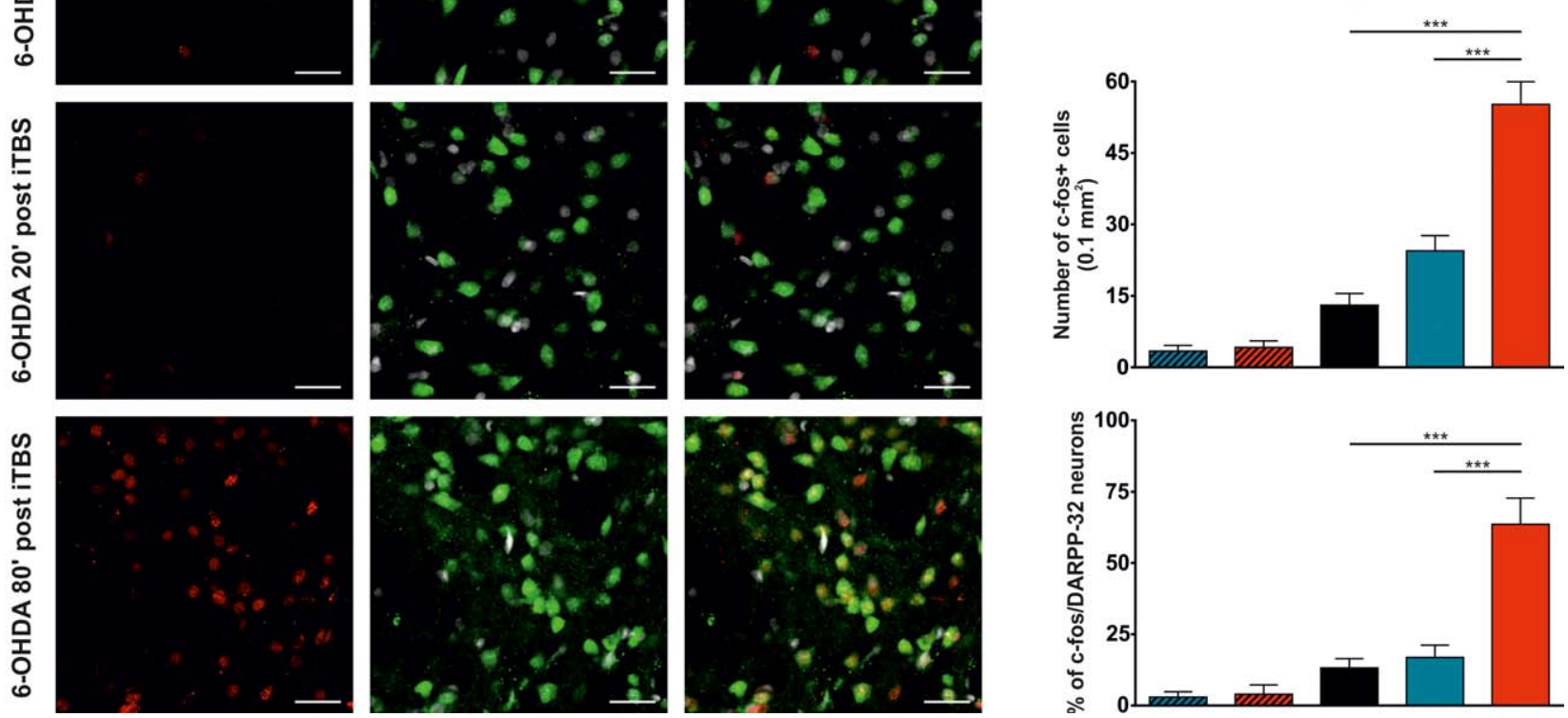

FIG. 4. A single session of intermittent theta-burst stimulation (iTBS) induces c-Fos activation in the striatum of 6-hydroxydopamine (6-OHDA) fully lesioned animals 80 minutes after treatment. a: Confocal images from dorsolateral striatum ipsilateral to the lesion side (schematic representation of the positioning on the upper right side) obtained from sham-operated (CTRL) 20 minutes and 80 minutes post-iTBS, 6-OHDA+sham-iTBS and 6OHDA animals 20 minutes and 80 minutes post-iTBS (12 samples per rat, $n=5$ animals per group). c-Fos is shown in red, dopamine- and cAMPregulated neuronal phosphoprotein (DARPP-32) in green, and 4',6-Diamidine-2'-phenylindole dihydrochloride (DAPI) in gray. In both CTRL conditions, only some c-Fos + cells colocalized with DARPP-32 (upper panels). In 6-OHDA animals, a slight increment in the number of colocalizing cells is visible (middle panel) as well as in 6-OHDA 20 minutes post-iTBS (4th panel). 6-OHDA 80 minutes post-iTBS animals showed a great number of c-Fos positive cells. Double immunofluorescence of c-Fos and DARPP-32 confirmed that c-Fos was mainly expressed by DARPP-32 medium spiny neurons (5th panel). Scale bars: $50 \mu \mathrm{m}$. b: Confocal images of c-Fos and choline acetyltransferase (ChAT) immunostaining in the ipsilateral striatum of 6-OHDA animals 80 minutes post-iTBS (upper panel) showed a lack of localization of c- Fos in cholinergic interneurons. Confocal images of cFos and parvalbumin (PV) immunostaining (lower panel) showed a lack of localization in parvalbumin-positive interneurons. Scale bars: $25 \mu \mathrm{m}$. c: Representative histogram of the total number of c-Fos positive cells (upper panel) and of the percentage of c-Fos + neurons colocalizing with DARPP-32 (lower panel). The 6-OHDA 80 minutes post-iTBS rats showed a marked increase in both total number of c-Fos + cells (1-way analysis of variance: treatment effect, $F_{4,20}=60.81, P<.001 ; 6-$ OHDA 80 minutes post-iTBS vs 6 -OHDA+sham-iTBS and 6 -OHDA 20 minutes post-iTBS: Bonferroni post hoc, $\left.{ }^{* \star} P<.001\right)$ and DARPP-32 colocalization (1-way analysis of variance: treatment effect, $F_{4,20}=26.28, P<.001 ; 6-\mathrm{OHDA} 80$ minutes post-iTBS vs all other groups: Bonferroni post hoc, $\left.{ }^{\star \star \star} P<.001\right)$. [Color figure can be viewed at wileyonlinelibrary.com] 
a

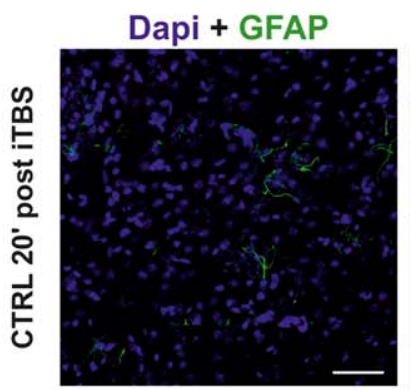

Dapi + Iba-1
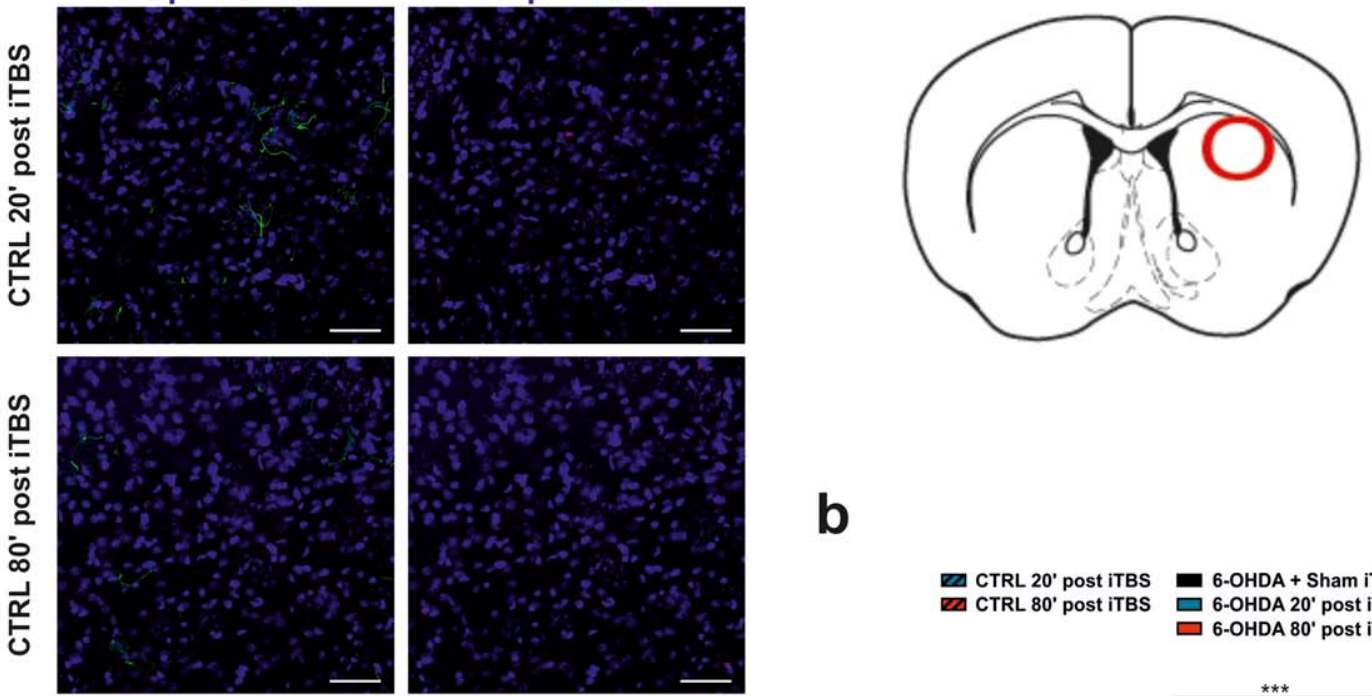

b
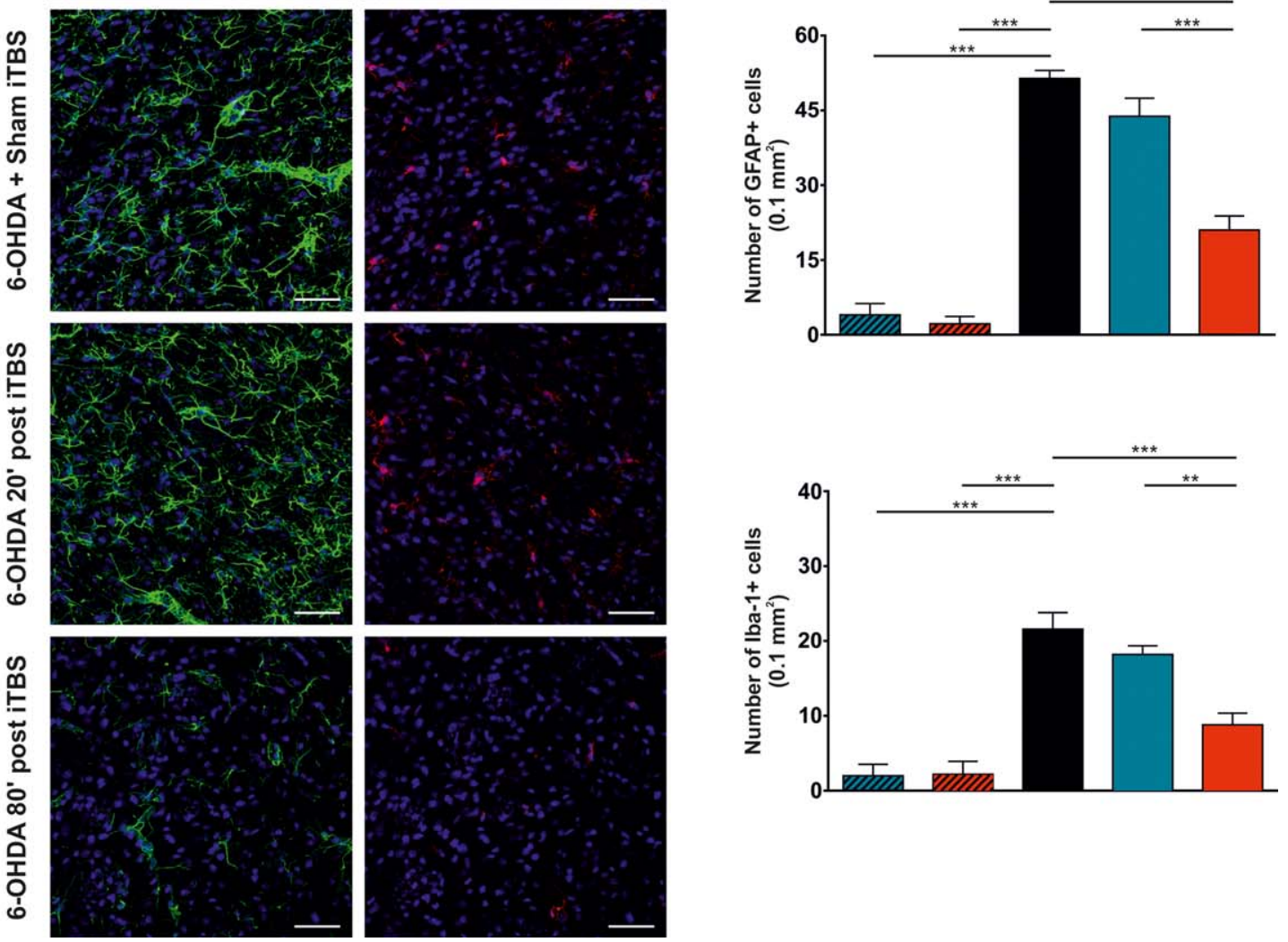

FIG. 5. Acute intermittent theta-burst stimulation (iTBS) reduces glial activation in the striatum of 6-hydroxydopamine (6-OHDA) fully lesioned animals 80 minutes after treatment. a: Confocal images from the ipsilateral striatum (dorso-lateral) of sham-operated (CTRL) 20 minutes and 80 minutes post-iTBS, 6-OHDA+sham-iTBS and 6-OHDA animals 20 minutes and 80 minutes post-iTBS reacted with Glial fibrillary acidic protein (GFAP) (green) and ionized calcium binding adaptor molecule 1 (lba-1) (red) and counterstained with 4',6-Diamidine-2'-phenylindole dihydrochloride (DAPI) (blue) to elucidate astrocytes (GFAP) and microglial (Iba-1) responses to iTBS (12 samples per rat, $n=5$ animals per group). iTBS treated sham-operated animals show little glial activation (upper panels). A state of neuroinflammation is evident in 6-OHDA animals, as expected (middle panel), and is still ongoing 20 minutes post-iTBS (4th panel). A striking reduction of neuroinflammation, in the form of a diminished number of both astrocytes and microglial cells, happened in 6-OHDA 80 minutes post-iTBS animals (bottom panel). Scale bars: $50 \mu \mathrm{m}$. b: Histogram of the total number of GFAP positive (upper panel) and Iba-1 positive cells (lower panel) in all groups. Data are reported as means \pm standard error of the mean. The 6OHDA+sham-iTBS animals show a very high astrocytic (1-way analysis of variance: treatment effect, $\left.F_{5,24}=91.26, P<.001\right)$ and microglial $(1$-way analysis of variance: treatment effect, $F_{5,24}=46.46, P<.001$ ) activation, compared to sham-operated animals (vs CTRL 20 minutes and 80 minutes post-iTBS: Bonferroni post hoc, ${ }^{* \star *} P<.001$ ). Inflammation is greatly reduced in $6-O H D A 80$ minutes post-iTBS rats as both the number of GFAP + cells and of Iba- $1+$ cells are reduced (GFAP + vs 6-OHDA+sham-iTBS: Bonferroni post hoc, ${ }^{\star \star *} P<.001$; Iba- $1+$ vs $6-\mathrm{OHDA}+$ sham-iTBS: Bonferroni post hoc, ${ }^{\star \star *} P<$.001). [Color figure can be viewed at wileyonlinelibrary.com] 
forelimb akinesia and asymmetry that are typically related to unilateral 6-OHDA lesion. A general increase of motor activity was observed in both paws in lesioned and sham-operated rats, according to the excitatory effect that this protocol exerts on motor cortical areas. However, when motor behavior was tested 80 minutes after a single iTBS session, a net beneficial effect was observed, resulting in similar limb use between ipsilateral and contralateral paws. In addition, gait abnormalities, consisting of a significant decrease in the regularity index, were attenuated 80 minutes after iTBS treatment in hemiparkisonian rats, which showed more organized step sequence patterns, resembling sham-operated rats.

From these data three basic questions emerged. The first asked if the increase in striatal DA was sufficient to rescue corticostriatal plasticity and function, the second dealt with the cell-type specificity of this response, and the third was related to the possible mechanisms underlying the therapeutic effects.

To address the first question, electrophysiological analysis was performed in SPNs by means of intracellular and patch-clamp recordings. Impairment in the induction of the two forms of corticostriatal plasticity, LTD and LTP, has been found to correlate with DA depletion and the onset of symptoms in experimental parkinsonism. In early PD, DA depletion initially does not affect LTD, but turns LTP into a form of shortterm plasticity. ${ }^{18}$ Later, when symptoms are fully manifested, both LTD and LTP are impaired. In our experiments, iTBS rescues both forms of synaptic plasticity in a temporal dynamic that reflects DA levels changes, suggesting that compensative mechanisms allowing LTP resurgence may depend on DA mechanisms more than that required by LTD. It is noteworthy that the time course of DA changes in response to TMS was different in the CTRL animals. A possible explanation is that in sham-operated animals, DA increase may rely on intact nigrostriatal terminals. In the parkinsonian condition, where the terminals are functionally absent, we suppose that an alternative source of DA may be engaged. Interestingly, after 20 minutes LTD is recovered, but such a return of function was not sufficient to allow a full recovery of LTP, providing a pattern of alterations similar to what observed in partially lesioned animals, modeling early symptomatic PD. ${ }^{18}$ This effect can be explained by the evidence that the induction of LTD depends on the activation of both $\mathrm{D} 1$ and $\mathrm{D} 2$ dopaminergic receptors, the latter being more sensitive to low levels of DA when compared with D1. However, coincident with a recovery of LTD at 20 minutes, a trough in DA levels was found. This finding seems to suggest that this effect may not depend on DA because this form of plasticity is under control of additional mechanisms, recruiting interneurons, and other signaling pathways ${ }^{30,31}$ all aimed at the preservation of SPNs activity and integrity. We therefore cannot rule out the possibility that an early compensative response of striatal circuitry allowing LTD recovery may recruit non-DA mechanisms. On this line, it has been reported that iTBS is able to induce long-term changes in the expression of activity-dependent proteins of the cortical inhibitory interneurons ${ }^{8}$ and in particular in the neuronal activity of inhibitory interneurons. ${ }^{32}$ Such selective activation of neuronal subtypes suggests that distinct TMS patterns can differentially affect specific neuronal populations.

To gain further insights into possible cell-type specific effects of iTBS in the dorsolateral striatum, electrophysiology was paralleled by the immunohistochemical characterization of c-Fos expression in the principal populations of striatal cells in 6-OHDA-lesioned rats. A modest increase of c-Fos immunoreactivity was found selectively in DARPP-32-expressing SPNs 20 minutes post-stimulation. When the experiments were replicated 80 minutes post-iTBS, the expression of corticostriatal LTP was fully recovered in the hemiparkinsonian rats. At the same time, c-Fos expression was greatly enhanced and the effect was restricted to DARPP-32-expressing neurons with no signal detected in interneurons. Concurrent analysis in the $\mathrm{SN}$ pars reticulata, motor thalamus, and motor cortex returned no changes in the early gene expression, clearly suggesting that iTBS has a cell typespecific and region-specific effect, coincident with the area in which DA increase was observed. In future studies it would be informative to analyze how this compensative changes vary with time by measuring c-Fos expression at different time points after TMS. Further discussion on this point may be found in the supplementary data file.

Such a selective effect despite an extensive stimulation points to the translational relevance of our results. In fact, it is possible that iTBS, by increasing DA in a particularly vulnerable but highly responsive area, thus restoring plasticity and motor control, may open a therapeutically relevant window in which functional and structural changes may reorganize the ability of striatal microcircuit to manage DA levels.

To test this hypothesis and to address the third question we studied two cellular populations that provide an indispensable support to neuronal activity by modulating DA and glutamate levels thus influencing synaptic plasticity: astrocytes and microglia. Recent evidence points to the ability of TMS to modulate the activity of glial cells, which have extensive connectivity and cell type-specific ability to directly or indirectly respond to neuronal firing, making them optimal candidates for mediating widespread TMS effects. ${ }^{33}$ Immunohistochemical analysis shows that in 6OHDA-lesioned rats, neurodegeneration progresses with time as a marked astrocytosis and microglial 
activation was strikingly visible two months after the lesion in the striatal samples. iTBS, as early as 20 minutes post-treatment, was able to reduce GFAP and Iba-1 immunoreactivity, but the greatest effect was found at 80 minutes, when the signal of both markers was lowered to control levels. These data are consistent with several studies recently reviewed by Cullen and Young, ${ }^{33}$ demonstrating abnormal microglia activation in postmortem samples from PD patients and in experimental parkinsonism. ${ }^{34-37}$ Microglia is implicated in the production of neurotrophins, interleukines, and proinflammatory and antiinflammatory citokines $^{34-37}$ that, if dysregulated, may contribute to abnormal plasticity. Also the finding of astrocytic changes in shape and functions in association with DA levels is supported by studies demonstrating that these cells in critical conditions release DA and express enzymes and the complete machinery to metabolize it. ${ }^{38-40}$ Moreover, relevant to PD, astrocytes can manage striatal DA levels, working as a reservoir for circulating levodopa. ${ }^{41}$

On this view, a different time course in sham- versus 6-OHDA-lesioned rats might reflect different mechanisms (synaptic vs nonsynaptic) by which DA levels change in response to TMS. Taken together, these data suggest that glial cells may have a role in iTBS effects on corticostriatal plasticity. Our results, if confirmed in PD patients, might be relevant in a therapeutic framework. Such intrinsic potential of the striatal synapse to implement the management of endogenous DA may offer an optimized response to DAreplacement therapies or to noninvasive therapeutic neuromodulation techniques.

Acknowledgments: This work was supported by grants from Fondazione Cariplo (2014-0660 to P.C.) and from the Italian Ministry of Health, Ricerca Finalizzata, and Giovani Ricercatori (GR-2010-2316671 to V.G., GR-2010.231052 to M.T.V., RF-2013-02357386 to B.P., and RF-2013-02356215 to P.C.).

\section{References}

1. Hirsch EC, Hunot S. Neuroinflammation in Parkinson's disease: a target for neuroprotection? Lancet Neurol 2009;8(4):382-397.

2. Barcia C. Glial-mediated inflammation underlying parkinsonism. Scientifica (Cairo) 2013;2013:357805.

3. Collins LM, Toulouse A, Connor TJ, Nolan YM. Contributions of central and systemic inflammation to the pathophysiology of Parkinson's disease. Neuropharmacology 2012;62(7):2154-2168.

4. Rossi S, Hallett M, Rossini PM, Pascual-Leone A, Safety of TMSCG. Safety, ethical considerations, and application guidelines for the use of transcranial magnetic stimulation in clinical practice and research. Clin Neurophysiol 2009;120(12):2008-2039.

5. Huang YZ, Edwards MJ, Rounis E, Bhatia KP, Rothwell JC. Theta burst stimulation of the human motor cortex. Neuron 2005;45(2): 201-206.

6. Benninger DH, Berman BD, Houdayer E, et al. Intermittent thetaburst transcranial magnetic stimulation for treatment of Parkinson disease. Neurology; 76(7):601-609.

7. Suppa A, Marsili L, Belvisi D, et al. Lack of LTP-like plasticity in primary motor cortex in Parkinson's disease. Exp Neurol; 227(2): 296-301.
8. Trippe J, Mix A, Aydin-Abidin S, Funke K, Benali A. Theta burst and conventional low-frequency rTMS differentially affect GABAergic neurotransmission in the rat cortex. Exp Brain Res 2009;199(3-4):411-421.

9. Benali A, Trippe J, Weiler E, et al. Theta-burst transcranial magnetic stimulation alters cortical inhibition. J Neurosci 2011;31(4): 1193-1203.

10. Calabresi P, Pisani A, Rothwell J, Ghiglieri V, Obeso JA, Picconi B. Hyperkinetic disorders and loss of synaptic downscaling. Nat Neurosci 2016;19(7):868-875.

11. Picconi B, Centonze D, Hakansson K, et al. Loss of bidirectional striatal synaptic plasticity in L-DOPA-induced dyskinesia. Nat Neurosci 2003;6(5):501-506.

12. Strafella AP, Paus T, Fraraccio M, Dagher A. Striatal dopamine release induced by repetitive transcranial magnetic stimulation of the human motor cortex. Brain 2003;126(Pt 12):2609-2615.

13. Khedr EM, Rothwell JC, Shawky OA, Ahmed MA, Foly N, Hamdy A. Dopamine levels after repetitive transcranial magnetic stimulation of motor cortex in patients with Parkinson's disease: preliminary results. Mov Disord 2007;22(7):1046-1050.

14. Keck ME, Welt T, Muller MB, et al. Repetitive transcranial magnetic stimulation increases the release of dopamine in the mesolimbic and mesostriatal system. Neuropharmacology 2002;43(1):101-109.

15. Kanno M, Matsumoto M, Togashi H, Yoshioka M, Mano Y. Effects of acute repetitive transcranial magnetic stimulation on dopamine release in the rat dorsolateral striatum. J Neurol Sci 2004;217(1):73-81.

16. Bagetta V, Sgobio C, Pendolino V, et al. Rebalance of striatal NMDA/AMPA receptor ratio underlies the reduced emergence of dyskinesia during D2-like dopamine agonist treatment in experimental Parkinson's disease. J Neurosci 2012;32(49):17921-17931.

17. Ghiglieri V, Pendolino V, Sgobio C, Bagetta V, Picconi B, Calabresi P. Theta-burst stimulation and striatal plasticity in experimental parkinsonism. Exp Neurol 2012;236(2):395-398.

18. Paille V, Picconi B, Bagetta V, et al. Distinct levels of dopamine denervation differentially alter striatal synaptic plasticity and NMDA receptor subunit composition. J Neurosci 2010;30(42):14182-14193.

19. Koopmans GC, Deumens R, Honig WM, Hamers FP, Steinbusch HW, Joosten EA. The assessment of locomotor function in spinal cord injured rats: the importance of objective analysis of coordination. J Neurotrauma 2005;22(2):214-225.

20. Pascucci T, Ventura R, Latagliata EC, Cabib S, Puglisi-Allegra S. The medial prefrontal cortex determines the accumbens dopamine response to stress through the opposing influences of norepinephrine and dopamine. Cereb Cortex 2007;17(12):2796-2804.

21. Westerink BH, Enrico P, Feimann J, De Vries JB. The pharmacology of mesocortical dopamine neurons: a dual-probe microdialysis study in the ventral tegmental area and prefrontal cortex of the rat brain. J Pharmacol Exp Ther 1998;285(1):143-154.

22. Cerovic M, Bagetta V, Pendolino V, et al. Derangement of Rasguanine nucleotide-releasing factor 1 (Ras-GRF1) and extracellular signal-regulated kinase (ERK) dependent striatal plasticity in LDOPA-induced dyskinesia. Biol Psychiatry 2015;77(2):106-115.

23. Calabresi P, Pisani A, Mercuri NB, Bernardi G. Long-term potentiation in the striatum is unmasked by removing the voltagedependent magnesium block of NMDA receptor channels. Eur J Neurosci 1992;4(10):929-935.

24. Calabresi P, Maj R, Pisani A, Mercuri NB, Bernardi G. Long-term synaptic depression in the striatum: physiological and pharmacological characterization. J Neurosci 1992;12(11):4224-4233.

25. Sasso V, Bisicchia E, Latini L, et al. Repetitive transcranial magnetic stimulation reduces remote apoptotic cell death and inflammation after focal brain injury. J Neuroinflammation 2016;13(1): 150.

26. Zangen A, Hyodo K. Transcranial magnetic stimulation induces increases in extracellular levels of dopamine and glutamate in the nucleus accumbens. Neuroreport 2002;13(18):2401-2405.

27. Cho SS, Strafella AP. rTMS of the left dorsolateral prefrontal cortex modulates dopamine release in the ipsilateral anterior cingulate cortex and orbitofrontal cortex. PLoS One 2009;4(8):e6725.

28. Strafella AP, Paus T, Barrett J, Dagher A. Repetitive transcranial magnetic stimulation of the human prefrontal cortex induces dopamine release in the caudate nucleus. J Neurosci 2001;21(15): RC157. 
29. Cardenas-Morales L, Volz LJ, Michely J, et al. Network connectivity and individual responses to brain stimulation in the human motor system. Cereb Cortex 2014;24(7):1697-1707.

30. Wang Z, Kai L, Day M, et al. Dopaminergic control of corticostriatal long-term synaptic depression in medium spiny neurons is mediated by cholinergic interneurons. Neuron 2006;50(3):443-452.

31. Yin HH, Davis MI, Ronesi JA, Lovinger DM. The role of protein synthesis in striatal long-term depression. J Neurosci 2006;26(46): 11811-11820.

32. Benali A, Trippe J, Weiler E, et al. Theta-burst transcranial magnetic stimulation alters cortical inhibition. J Neurosci 2011;31(4): 1193-1203.

33. Cullen CL, Young KM. How does transcranial magnetic stimulation influence glial cells in the central nervous system? Front Neural Circuits 2016;10:26.

34. Cicchetti F, Brownell AL, Williams K, Chen YI, Livni E, Isacson O. Neuroinflammation of the nigrostriatal pathway during progressive 6-OHDA dopamine degeneration in rats monitored by immunohistochemistry and PET imaging. Eur J Neurosci 2002; 15(6):991-998.

35. McGeer PL, McGeer EG. Glial cell reactions in neurodegenerative diseases: pathophysiology and therapeutic interventions. Alzheimer Dis Assoc Disord 1998;12(suppl 2):S1-S6.

36. McGeer PL, Schwab C, Parent A, Doudet D. Presence of reactive microglia in monkey substantia nigra years after 1-methyl-4- phenyl-1,2,3,6-tetrahydropyridine administration. Ann Neurol 2003;54(5):599-604.

37. Orr CF, Rowe DB, Mizuno Y, Mori H, Halliday GM. A possible role for humoral immunity in the pathogenesis of Parkinson's disease. Brain 2005;128(Pt 11):2665-2674.

38. Inyushin MY, Huertas A, Kucheryavykh YV, et al. L-DOPA uptake in astrocytic endfeet enwrapping blood vessels in rat brain. Parkinsons Dis 2012;2012:321406.

39. Oliva I, Fernandez M, Martin ED. Dopamine release regulation by astrocytes during cerebral ischemia. Neurobiol Dis 2013;58:231241.

40. Tsai MJ, Lee EH. Characterization of L-DOPA transport in cultured rat and mouse astrocytes. J Neurosci Res 1996;43(4):490495 .

41. Asanuma M, Miyazaki I, Murakami S, Diaz-Corrales FJ, Ogawa N. Striatal astrocytes act as a reservoir for L-DOPA. PLoS One 2014;9(9):e106362.

\section{Supporting Data}

Additional Supporting Information may be found in the online version of this article at the publisher's web-site. 